\title{
Artificial intelligence and data processing in injury diagnosis and prevention in competitive sports: $A$ literature review
}

\begin{abstract}
Artificial intelligence (AI) application opens an exciting perspective for predicting injury risk and team sports performance. A better understanding of the techniques of AI employed and of the sports that are using AI is warranted. The purpose of this study is to identify which AI approaches have been applied to investigate sports performance and injury risk
\end{abstract}

Keywords: Artificial intelligence, fuzzy logic, diagnosis, prevention, sports
Volume I3 Issue 2 - 202 I

\author{
Panagiotis Poulios, Athanasios Serlis, Peter P \\ Groumpos, loannis Gliatis \\ 'Barnet Hospital, Department of Orthopaedic, Royal Free NHS \\ Trust, United Kingdom \\ ${ }^{2}$ Royal London Hospital, Department of Orthopaedics, Barts \\ NHS Trust, United Kingdom \\ ${ }^{3}$ Professor, Department of Electrical and Computer Engineering, \\ University of Patras, Greece \\ ${ }^{4}$ Associate Professor, Department Orthopaedic, University of \\ Patras, Greece
}

Correspondence: Panagiotis Poulios, Barnet Hospital,

Orthopaedic Department, Royal Free NHS Trust, Wellhouse Ln, Barnet EN5 3DJ, United Kingdom, Tel 07378482272 ,

Email panagiotis.poulios@nhs.net

Received: May 04, 202I | Published: May 14, 202 I

\section{Introduction}

Medical diagnosis involves complex problem-solving situations because there are complex interrelation and interaction between the human body and mind (physiology and psychology). There is still a fuzzy knowledge of how the human body works, especially in sports. ${ }^{1}$ More precisely, Tirabassi et al. ${ }^{2}$ claim that medically disqualifying injuries vary in gender, age, sport, and athletic activity type. Also, Patel et al. ${ }^{3}$ mention that intense participation and specialisation in sports is a dominant trend today, even for the early ages of one's life, leading to new and complicated sport-related musculoskeletal injuries. Injuries are determinant factors of success and lost revenues, and for this reason, it is of great importance to accurately and precisely prevent and diagnose injuries and their risk. ${ }^{4}$

Further, one of the main challenges in sport is the increase in athletes' performance and injuries prevention. For this reason, accuracy in the decision-making process in injuries' prevention and diagnosis is necessary. ${ }^{5}$ Within this framework, technology is essential. ${ }^{6}$

Artificial intelligence methodology can help in situations where the decision should be made in complex problems, such as sport medical diagnosis. Artificial intelligence is considered a tool for supporting quick, consistent, and precise diagnoses with limited and unclear information (Innocent et al., 2004). Since artificial intelligence offers many potentials to predicting and preventing injuries and risk of injuries in sports medicine, it is of great interest to examine the use of this technology to better understand its dynamic and future perspectives. The present paper scope is to present data concerning the application of artificial intelligence in this field.

\section{Literature review}

Technological advancements have helped in complicated medical diagnosis. For instance, Knudson ${ }^{7}$ refers to the domain of kinesiology, arguing that the advances in computational neuroscience and imaging can help in better understanding the interrelation between physical activity and cognitive function. The author also contends that computing and modelling advances allow for simulations of complex systems, for example, the human body. Also, Seshadri et al. ${ }^{8}$ refers to the biomedical wearable technologies in the field of sports medicine, where the wireless technology can help in tracking and monitoring physiological parameters, enabling better athletes' care, which in turn can be used in the creation of predictive models, based on the data collected, for the design of athletes' treatment plans. Similarly, Adesida et al. ${ }^{9}$ refer to the advantages of wearable technology in sport kinematics and kinetics in injury prevention.

In general, data mining methods within the artificial intelligence that have been reported in sports medicine, and in injury prediction among others, are: ${ }^{10}$ artificial neural networks, support vector machines, decision trees, fuzzy systems, Bayesian methods, and logistic regressions. For example, the fuzzy sets approach, introduced in 1965, has been applied in various fields, and sport medical decisions, among others. ${ }^{11}$ Artificial neural networks have been used in predicting the risk of sport-related injury. ${ }^{4}$

\section{The use of fuzzy sets}

Even from the previous decade researchers examined how fuzzy systems can help in the multifaceted decision-making process. Innocent et al. ${ }^{1}$ reviewed the case of fuzzy systems for medical diagnosis. In their paper, the authors referred to the categorical consistency, which can help achieve repeatable classification performance. Categorical consistency is a requirement for consultation systems, where one should be able to classify the same situation in the same category in a repeated way. The creation of consistent groups for classification is based upon intelligent processing, which allows the re-consideration of the classification schemes, as in sports injuries and different 
patients' cases. In this complex situation, fuzzy neural networks can understand and facilitate the classification procedure. The system developed by Inoccent et al. ${ }^{1}$ is based on the extraction of features from bone scan (radiographic) images that were later analysed through a clustering algorithm, within the framework of Adaptive Resonance Theory (ART). This resulted in the creation of the ART fuzzy neuro-clustering technique. The authors concluded that vague representation and the fuzzy neural clustering methods had improved the radiographic images' classification process, even though there is intrinsic vagueness. However, these two studies do not refer to sports medicine; hence, more research in the application of these techniques in the prevention and diagnosis of the sport-related injury is vital.

Similarly, Hung ${ }^{11}$ refers to medical pattern recognition, arguing that fuzzy approaches can help classify and identify patterns between the observed unknown samples and the known samples. The author developed the so-called intuitionistic fuzzy likelihood-based Measurement (IFLM), which can help physicians in their preliminary medical diagnosis with accuracy, considering the uncertainty in the decision-making process. Within the context of fuzzy sets, Wang, Dai, Chen and Meng ${ }^{12}$ developed a new approach, the so-called intuitionistic fuzzy evidential reasoning (IFER), helping physicians improve their accuracy medical diagnosis. Das et al. ${ }^{13}$ integrated fuzzy and intuitionistic fuzzy frameworks to develop a web-based medical diagnostic support system. Fuzzy logic was valuable in a complex medical framework with increased uncertainty since it can treat medical data as fuzzy sets. The authors concluded that the model developed has several benefits and can be used as diagnostic tools.

\section{The use of artificial neural networks}

Dower et al. ${ }^{4}$ refer to algorithms that help estimate the risk of injuries in the case of sports. For example, there is a metric called Acute Chronic Workload Ratio (ACWR): Still, its disadvantage is that it cannot provide precise and accurate estimation when used in isolation. The authors propose the analytical engine Injury Risk Mitigation System (IRMS), based upon various time-series data (e.g. GPS volume metric) and static data (e.g. age, injury history) for the estimation of the injury risk using machine learning (artificial neural network). The artificial neural networks that can be used in the artificial intelligence of the IRMS system are Feedforward Neural Networks (FFNN), Recurrent Neural Networks (RNN) and Convolutional Neural Networks $(\mathrm{CNN})$. The inputs used in this system lead to information related to a potential injury-event, which constitutes the building blocks upon which an artificial intelligence application is built. Overall, the IRMS system can help identify the training regime patterns related to injuries, assisting in predicting the probability of occurring future injuries in a training plan, using historical time series data and the training plan.

Jaspers et al. ${ }^{14}$ used in their research an artificial neural network (2018) to examine the association between external and internal training load in athletes of professional soccer. This study aimed to investigate the prediction of perceived exertion in this sport, which may be used to prevent injuries. This study indicated that artificial neural networks could be successfully used to predict the training load, and thus for load monitoring in soccer athletes. Similarly, Bartlett et al. ${ }^{15}$ used artificial neural networks to examine their added value in predicting perceived exertion from training load variables in team sport athletes. Their study concluded that machine learning approaches might perform better compared to more traditional methods. Thus, they are promising in accurate training prescription and evaluation, hence avoiding overtraining and injury.

The use of artificial neural networks, along with electrocardiography, was found successful and promising in detecting heart defects among athletes in the study of Adetiba et al. ${ }^{16}$ In the study of Qilin et al. ${ }^{17}$ utilised artificial neural networks to predict knee joint injury in college football training, and it was found that the model could successfully identify the factors that can lead to injuries of the knee joint. Michałowska et al. ${ }^{18}$ used artificial neural networks in knee injury risk evaluation among professional football players. The results of their study indicated that neural networks could be a useful diagnostic tool in the case of evaluating risk injury, under the condition of the appropriate database for artificial neural network training.

\section{The use of other models}

The study of Whiteside et al. ${ }^{19}$ examined the use of naïve Bayes and support vector machine to predict factors in ulnar collateral ligament reconstruction surgeries in the case of players in Major League Baseball. The model successfully classified $66.8 \%$ of patients and identified six primary elements. Overall, this study's results indicate that machine learning models can improve the prevention of UCL reconstruction surgery in baseball players. Bayesian regulated neural networks, along with Gaussian distributions, were used in the study of Ertelt, et al. ${ }^{20}$ to examine ground reaction force patterns in a sample of male athletes from different sports. The model successfully classified $94.29 \%$ of cases, leading to a promising and adequate method for classifying forced patterns. Bayesian classification and Assistant algorithm for top-down induction of decision trees classifier as artificial intelligence tools were used from Zelic et al. ${ }^{21}$ to diagnose sports injuries. The model was not successful initially, and for this reason, the authors added expert-defined diagnostic rules as preclassifiers. The authors proposed the final model that the Bayesian classifier with the fuzzy discretisation of numerical attributes was accurate, and for this reason, as a promising machine learning tool that can help physicians in their decision-making process. Lu et al. ${ }^{22}$ used Bayesian theory, likelihood ratios, and predictive data mining to improve the precision in the diagnosis of rotator cuff tears. The authors concluded that this model could be a useful tool in classification and the diagnosis of rotator cuff tears.

The use of EMG vectors was examined in the study of Malfait et $a .^{23}$ in the case of jump landing activities, to identify potential associations between neuromuscular activation patterns and landing kinematics multidirectional sports (e.g. volleyball and handball), to determine possible risk injury. The results were promising for the use of EMG vectors. Various machine learning approaches were examined in the study of López-Valenciano et al. ${ }^{24}$ to identify the best-performing injury risk factor model to identify athletes at risk for lower extremity muscle injuries case of professional handball and soccer players. The authors concluded that the best model was the model generated by the Smoote Boost technique. Rossi et al. ${ }^{25}$ developed an artificial intelligence multi-dimensional model based on machine learning and GPS training data to forecast soccer players' injuries. The model proposed it was both interpretable and accurate, leading to promises in the field of injury prevention in soccer players and interpreting the relation between training and injury. The performance of various artificial intelligence tools, with an emphasis upon artificial neural networks, was examined in weight training from 
Novatchkov and Baca. ${ }^{26}$ The study results indicated that artificial intelligence could assess and help in the classification in the case of weight training, which can help in the prevention of sports injuries.

\section{Future challenges}

One possible future trend in applying artificial intelligence in sports medicine is related to crowd innovation, along with the use of artificial intelligence. As Mak et al. ${ }^{27}$ argue, "crowd innovation has been successfully applied to a variety of genomic and computational biology problems by using prize-based competitions to identify extreme value solutions that outperform those developed by conventional academic approaches, whereas "artificial intelligence has been successfully applied to diagnostic subspecialties of medicine". The authors implemented a three-phase, prize-based crowd innovation program of ten weeks, where 34 contestants submitted 45 algorithms, identified multiple AI solutions in the case of lung tumours within the context of radiation therapy. The authors concluded that online content could help address unmet needs and apply new skills in medicine's artificial intelligence methods. This program can also be implemented in sports medicine, to create new ways for preventing and diagnosing injuries and the risk of injury in the case of athletes.

Artificial intelligence applies machine learning techniques that can help physicians in complex decision-making processes, especially in risk predictions and prevention, based upon the power of computers to make predictions and model patterns, through learning by their associations. However, this computational intelligence comes with some limitations, concerning two significant areas: a) bias concerning the interpretation of the data and data inputs to better understand the quantified benefit for athletes; b) ethical dilemmas concerning legislation and athlete-related information. ${ }^{28}$

Also, more empirical research is needed to examine the performance of specific artificial intelligence techniques. This is based on the fact that there are studies where these techniques have poor performance. For instance, Ruddy et al. ${ }^{29}$ used various machine learning techniques and the Bayesian network to predict hamstring strain injury in elite Australian footballers. However, according to their conclusions, these models were characterised by poor predictive performance. Hence, the authors do not recommend the use of artificial intelligence techniques. In another study, the study of Taylor et $\mathrm{al}^{30}$ artificial intelligence integrated into a combination of ground reaction force patterns and biomechanics (screening) was used in risk injury assessment in the case of activities such as jump landing in female athletes playing multidirectional sports. This study aimed to examine the extent to which lower extremity biomechanics measured during a jump landing on a double leg after a sagittal plane movement is representative of biomechanics measured during single-leg or frontal plane jump landing tasks. The results indicated that standard doubleleg sagittal plane jump landing tasks might not adequately represent the lower extremity biomechanics during single-leg activities. For this reason, more research is needed in the case of screening and preventing injury risk in neuromuscular injury prevention programs.

Finally, within the framework of computational intelligence, Fister, Ljubic, Suganthan, Perc and Fister (2015) argue that there is one more significant challenge that needs to be addressed and overcome: data mining. The authors conclude that sport research studies are based on data mining. However, the current data mining techniques are not sufficient and adequate in addressing the complexity of the sports medicine field. For this reason, Fister et al..$^{31}$ call for an artificial trainer, who should provide all the services provided by a human coach at a lower cost. For this artificial trainer to be effective, should incorporate the following features: clustering, classification, sequential pattern mining, and association rule mining. Furthermore, computational intelligence algorithms that can virtual simulations and detect factors related to injuries are also necessary.

\section{Conclusion}

The effective use of artificial intelligence in sports medicine in the prevention and diagnosis of injuries in athletes and the modelling and detection/estimation of risk injuries is still under development. Various researchers have developed the latest decades of multiple solutions, such as fuzzy sets, Markov process, and artificial neural networks. This paper tried to offer an insight into this domain, presenting some data concerning artificial intelligence tools and methods that have been developed. However, the major limitation of this study is that a more systematic review is needed. Further theoretical and above all, empirical research is required to examine this technology's benefits in sports medicine. More precisely, even though this presentation can indicate that artificial intelligence is promising, further evaluation research is needed, establishing the predictive and diagnosing performance of specific artificial intelligence tools and methods.

\section{Acknowledgments}

None.

\section{Conflicts of interest}

The authors declare no conflicts of interest.

\section{Funding}

None.

\section{References}

1. Innocent PR, John RI, Garibaldi JM. Fuzzy methods for medical diagnosis. Applied Artificial Intelligence. 2004;19(1):69-98.

2. Tirabassi J, Brou L, Khodaee M, et al. Epidemiology of High School Sports-Related Injuries Resulting in Medical Disqualification: 20052006 Through 2013-2014 Academic Years. The American Journal of Sports Medicine. 2016;44(11):2925-2932.

3. Patel DR, Yamasaki A, Brown K. Epidemiology of sportsrelated musculoskeletal injuries in young athletes in the United States. Translational Pediatric. 2017;6(3):160-166.

4. Dower C, Rafehi A, Weber J, et al. An enhanced metric of injury risk utilising Artificial Intelligence. MIT Sloan Sports Analytics. Conference. 2018 .

5. Lames M, Eskofier B, Schöllhorn W, et al. Working Groups. In: Lemmink K, Morgan S, Sampaio J, editors. Computer Science in HighPerformance Sport - Applications and Implications for Professional Coaching. Germany: Dagstuhl Reports. 2018. pp. 48-49.

6. Wu HJ, Zhao HY. Digital human technology and its application in the field of sports medicine and Prospect. Journal Of Applied Science And Engineering Innovation. 2014;1(1):49-53.

7. Knudson D. Future Trends in the Kinesiology Sciences. Quest. 2016;68(3):348-360.

8. Seshadri DR, Magliato S, Voos JE, et al. Clinical translation of biomedical sensors for sports medicine. J Med Eng Technol. 2019;43(1):66-81. 
9. Adesida Y, Papi E, McGregor AH. Exploring the Role of Wearable Technology in Sport Kinematics and Kinetics: A Systematic Review. Sensors. 2019;19(7):1597.

10. Haghighat M, Rastegari H, Nourafza N. A Review of Data Mining Techniques for Result Prediction in Sports. ACSIJ Advances in Computer Science: an International Journal. 2013;2(5):7-12.

11. Hung KC. Applications of medical information: Using an enhanced likelihood measured approach based on intuitionistic fuzzy sets. IIE Transactions on Healthcare Systems Engineering. 2012;2(3):224-231.

12. Wang Y, Dai Y, Chen Y, et al. The Evidential Reasoning Approach to Medical Diagnosis using Intuitionistic Fuzzy Dempster-Shafer Theory. International Journal of Computational Intelligence Systems. 2015;8(1):75-94.

13. Das S, Guha D, Dutta B. Medical diagnosis with the aid of using fuzzy logic and intuitionistic fuzzy logic. Applied Intelligence. 2016;45(3):850-867.

14. Jaspers A, De Beéck TO, Brink MS, et al. Relationships Between the External and Internal Training Load in Professional Soccer: What Can We Learn From Machine Learning? International Journal of Sports Physiology and Performance. 2018;13(5):625-630.

15. Bartlett JD, O'Connor F, Pitchford N, et al. Relationships between internal and external training load in team-sport athletes: evidence for an individualised approach. International Journal of Sports Physiology and Performance. 2017;12(2):230-234.

16. Adetiba E, Iweanya VC, Popoola SI, et al. Automated detection of heart defects in athletes based on electrocardiography and artificial neural network. Cogent Engineering, 2017;4.

17. Qilin S, Xiaomei W, Xiaoling F, et al. Study on knee joint injury in college football training based on artificial neural network. Revista Iberica de Sistemas e Tecnologias de Informacao. 2016;16:197-210.

18. Michałowska M, Walczak T, Grabski JK, et al. Artificial neural networks in knee injury risk evaluation among professional football players. AIP Conference Proceedings. 1922; 2018.

19. Whiteside D, Martini DN, Lepley AS, et al. Predictors of ulnar collatera ligament reconstruction in Major League Baseball pitchers. American Journal of Sports Medicine. 2016;44(9):2202-2209.
20. Ertelt T, Solomonovs I, Gronwald T. Enhancement of force patterns classification based on Gaussian distributions. Journal of Biomechanics. 2018;67:144-149.

21. Zelic I, Kononenko I, Lavrac N, et al. Induction of decision trees and Bayesian classification applied to the diagnosis of sports injuries. Journal of Medical Systems. 1997;21(6):429-444.

22. Lu HY, Huang CY, Su CT, et al. Predicting Rotator Cuff Tears Using Data Mining and Bayesian Likelihood Ratios. PLoS One. 2014;9(4).

23. Malfait B, Dingenen B, Smeets A, et al. Knee and Hip Joint Kinematics Predict Quadriceps and Hamstrings Neuromuscular Activation Patterns in Drop Jump Landings. PLoS One. 2016;11(4):e0153737.

24. López-Valenciano A, Ayala F, Puerta JM, et al. A Preventive Model for Muscle Injuries: A Novel Approach based on Learning Algorithms. Med Sci Sports Exerc. 2018;50(5):915-927.

25. Rossi A, Pappalardo L, Cintia P, et al. Effective injury forecasting in soccer with GPS training data and machine learning. PLoS One. 2018.

26. Novatchkov H, Baca A. Artificial Intelligence in Sports on the Example of Weight Training. J Sports Sci Med. 2013;12(1):27-37.

27. Mak RH, Endres MG, Paik JH, et al. Use of Crowd Innovation to Develop an Artificial Intelligence-Based Solution for Radiation Therapy Targeting. JAMA Oncology. 2019;5(5):654-661.

28. Challen R, Denny J, Pitt M, et al. Artificial intelligence, bias and clinical safety. BMJ Quality \& Safety. 2019;28(3):231-237.

29. Ruddy JD, Shield AJ, Maniar N, et al. Predictive modelling of hamstring strain injuries in elite Australian Footballers. Med Sci Sports Exerc. 2018;50(5):906-914.

30. Taylor JB, Ford KR, Nguyen AD, et al. Biomechanical Comparison of Single- and Double-Leg Jump Landings in the Sagittal and Frontal Plane. Orthopaedic Journal of Sports Medicine. 2016;4(6).

31. Fister I, Ljubic K, Suganthan P N, et al. Computational intelligence in sports: Challenges and opportunities within a new research domain. Applied Mathematics and Computation. 2015;262(1):178-186. 